\title{
Erratum: Strong decays of exotic and nonexotic heavy baryons in the chiral quark-soliton model [Phys. Rev. D 96, 094021 (2017)]
}

\author{
Hyun-Chul Kim, Maxim V. Polyakov, Michał Praszałowicz, and Ghil-Seok Yang
}

(Received 11 January 2018; published 12 February 2018)

\begin{abstract}
We present a corrected calculation of the decay widths of the putative exotic heavy $\Omega_{c}^{0}$ states to the ground state $\mathrm{SU}(3)_{\text {flavor }}$ sextets of heavy baryons. In the article we have used formulas summed over the final spin $1 / 2$ and $3 / 2$ sextets. These states, however, are degenerate only in the strict $m_{Q} \rightarrow \infty$ limit, and therefore for phenomenological applications one has to use formulas for fixed final state spin. The correct numerical values of the decay widths are only marginally affected and all conclusions remain unchanged.
\end{abstract}

DOI: 10.1103/PhysRevD.97.039901

In the article we have calculated the decay widths of heavy baryons in the framework of the chiral quark soliton model ( $\chi \mathrm{QSM})$ where a heavy baryon is a composite object built from the soliton of integer spin $S=0$ or 1 and a heavy quark of spin 1/2. Soliton spin depends on the $\mathrm{SU}(3)_{\text {flavor }}$ representation of the light quarks. For SU(3) flavor sextet and for exotic $\overline{\mathbf{1 5}}$ $S=1$, and heavy baryons come in degenerate spin $1 / 2$ and $3 / 2$ doublets. This degeneracy is lifted by the hyperfine spinspin interaction.

In the $\chi \mathrm{QSM}$ the decay operator takes the form of the product of the intrinsic operator describing the emission of the pseudoscalar meson $\varphi$ by the soliton, and the momentum of $\varphi$ denoted as $\vec{p}$,

$$
\mathcal{O}_{\varphi}=\sum_{m} \mathcal{O}_{\varphi, m} p_{m}
$$

The decay amplitude is calculated by sandwiching $\mathcal{O}_{\varphi}$ between the heavy baryon wave functions

$$
\mathcal{A}_{\varphi}=\left\langle B_{2}^{\mathcal{R}_{2}} J_{2} M_{2}\left|\mathcal{O}_{\varphi}\right| B_{1}^{\mathcal{R}_{1}} J_{1} M_{1}\right\rangle,
$$

where the wave function is a linear combination of a soliton state and a heavy quark state,

$$
\left|B^{\mathcal{R}} J M_{J}\right\rangle=\sum_{M, m}|1 / 2, M\rangle \otimes\left|(\mathcal{R}) B,\left(-Y^{\prime}, s, m\right)\right\rangle\left(\begin{array}{cc|c}
s & 1 / 2 & J \\
m & M & M_{J}
\end{array}\right) .
$$

Here $J, M_{J}$ correspond to the heavy baryon spin, $s, m$ to the soliton spin; $Y^{\prime}=2 / 3$ and $B^{\mathcal{R}}=\left(Y, T, T_{3}\right)$ are $\mathrm{SU}(3)$ quantum numbers for a baryon in representation $\mathcal{R}$. In order to calculate the width we have to square the amplitude and average over the initial spins and sum over the final spins with the following result:

$$
\overline{\mathcal{A}_{\varphi}^{2}}=\frac{1}{2 J_{1}+1} \sum_{M_{1}} \sum_{J_{2}, M_{2}} \mathcal{A}_{\varphi}^{2}=a_{\varphi}^{2} \frac{1}{3} p^{2},
$$

where we have explicitly displayed factor $1 / 3$ that comes from the spin summation and $a_{\varphi}$ is the amplitude that one obtains averaging the operator $\mathcal{O}_{\varphi}$ over the soliton rotational wave function. In practice, however, we do not perform summation over $J_{2}$ because the hyperfine split states are not degenerate. In the article we have erroneously used factor $1 / 3$ for all decays. In order to correct this, decay modes that involve hyperfine split baryons in the final state have to be multiplied by $\gamma=\gamma\left(J_{1} \rightarrow J_{2}\right)$ given below,

$$
\begin{array}{ll}
\gamma(1 / 2 \rightarrow 1 / 2)=2 / 3, & \gamma(1 / 2 \rightarrow 3 / 2)=1 / 3, \\
\gamma(3 / 2 \rightarrow 1 / 2)=1 / 6, & \gamma(3 / 2 \rightarrow 3 / 2)=5 / 6 .
\end{array}
$$


TABLE I. $\quad \Omega_{c}\left(\overline{\mathbf{1 5}}_{1}, 1 / 2\right)$ partial and total decay widths in MeV. Experimental value is from the LHCb measurement [2].

\begin{tabular}{llcr}
\hline \hline$\#$ & \multicolumn{1}{c}{ Decay } & This work & Experimental value \\
\hline & $\Omega_{c}\left(\overline{\mathbf{1 5}}_{1}, 1 / 2\right) \rightarrow \Xi_{c}\left(\overline{\mathbf{3}}_{0}, 1 / 2\right)+K$ & 0.339 & $\ldots$ \\
& $\Omega_{c}\left(\overline{\mathbf{1 5}}_{1}, 1 / 2\right) \rightarrow \Omega_{c}\left(\mathbf{6}_{1}, 1 / 2\right)+\pi$ & 0.065 & $\ldots$ \\
& $\Omega_{c}\left(\overline{\mathbf{1 5}}_{1}, 1 / 2\right) \rightarrow \Omega_{c}\left(\mathbf{6}_{1}, 3 / 2\right)+\pi$ & 0.015 & $\ldots$ \\
9 & Total & 0.42 & $0.8 \pm 0.2 \pm 0.1$ \\
\hline \hline
\end{tabular}

TABLE II. $\Omega_{c}\left(\overline{\mathbf{1 5}}_{1}, 3 / 2\right)$ partial and total decay widths in MeV. Experimental value is from the LHCb measurement [2].

\begin{tabular}{lccc}
\hline \hline$\#$ & Decay & This work & Experimental value \\
\hline & $\Omega_{c}\left(\overline{\mathbf{1 5}}_{1}, 3 / 2\right) \rightarrow \Xi_{c}\left(\overline{\mathbf{3}}_{0}, 1 / 2\right)+K$ & 0.848 & $\ldots$ \\
& $\Omega_{c}\left(\overline{\mathbf{1 5}}_{1}, 3 / 2\right) \rightarrow \Xi_{c}\left(\mathbf{6}_{1}, 1 / 2\right)+K$ & 0.002 & $\ldots$ \\
& $\Omega_{c}\left(\overline{\mathbf{1 5}}_{1}, 3 / 2\right) \rightarrow \Omega_{c}\left(\mathbf{6}_{1}, 1 / 2\right)+\pi$ & 0.028 & $\ldots$ \\
& $\Omega_{c}\left(\overline{\mathbf{1 5}}_{1}, 3 / 2\right) \rightarrow \Omega_{c}\left(\mathbf{6}_{1}, 3 / 2\right)+\pi$ & 0.080 & $\ldots$ \\
10 & Total & 0.97 & $1.1 \pm 0.8 \pm 0.4$ \\
\hline \hline
\end{tabular}

Note that $\sum_{J_{2}} \gamma\left(J_{1} \rightarrow J_{2}\right)=1$. For the decays to final states with soliton spin $S_{2}=0$ no reweighting factor is needed, since there are no hyperfine split states in this case.

With this change the results of the article have to be modified as follows [see Eq. (10) in the article]:

$$
\begin{aligned}
& \Gamma_{\Sigma\left(\mathbf{6}_{1}\right) \rightarrow \Lambda\left(\overline{\mathbf{3}}_{0}\right)+\pi}=\frac{1}{72 \pi} \frac{p^{3}}{F_{\pi}^{2}} \frac{M_{\Lambda\left(\overline{\mathbf{3}}_{0}\right)}}{M_{\Sigma\left(\mathbf{6}_{1}\right)}} H_{\overline{\mathbf{3}}}^{2} \frac{3}{8}, \\
& \Gamma_{\Xi\left(\mathbf{6}_{1}\right) \rightarrow \Xi\left(\overline{\mathbf{3}}_{0}\right)+\pi}=\frac{1}{72 \pi} \frac{p^{3}}{F_{\pi}^{2}} \frac{M_{\Xi\left(\overline{\mathbf{3}}_{0}\right)}}{M_{\Xi\left(\mathbf{6}_{1}\right)}} H_{\overline{\mathbf{3}}}^{2} \frac{9}{32}, \\
& \Gamma_{\Omega\left(\overline{\mathbf{1 5}}_{1}\right) \rightarrow \Xi\left(\overline{\mathbf{3}}_{0}\right)+K}=\frac{1}{72 \pi} \frac{p^{3}}{F_{K}^{2}} \frac{M_{\Xi\left(\overline{\mathbf{3}}_{0}\right)}}{M_{\Omega\left(\overline{\mathbf{1 5}}_{1}\right)}} G_{\overline{\mathbf{3}}}^{2} \frac{3}{10}, \\
& \Gamma_{\Omega\left(\overline{\mathbf{1 5}}_{1}\right) \rightarrow \Omega\left(\mathbf{6}_{1}\right)+\pi}=\frac{1}{72 \pi} \frac{p^{3}}{F_{\pi}^{2}} \frac{M_{\Omega\left(\mathbf{6}_{1}\right)}}{M_{\Omega\left(\overline{\mathbf{1 5}}_{1}\right)}} G_{\mathbf{6}}^{2} \frac{4}{15} \gamma, \\
& \Gamma_{\Omega\left(\overline{\mathbf{1 5}}_{1}\right) \rightarrow \Xi\left(\mathbf{6}_{1}\right)+K}=\frac{1}{72 \pi} \frac{p^{3}}{F_{K}^{2}} \frac{M_{\Xi\left(\mathbf{6}_{1}\right)}}{M_{\Omega\left(\overline{\mathbf{1 5}}_{1}\right)}} G_{\mathbf{6}}^{2} \frac{2}{15} \gamma .
\end{aligned}
$$

Note that this modification changes only the numerical results for the total widths of exotic baryons by at most $13 \%$ not affecting any conclusion of the article. However, for completeness we present below the corrected results for Tables III-IV of the article. We also show a modified Fig. 2 of the article.

The details of the calculations leading to the correction factors $\gamma$ are presented in the appendix.

TABLE III. Predictions in MeV for the partial and total decay widths of explicitly exotic $\Xi_{c}^{3 / 2}\left(\overline{\mathbf{1 5}}_{1}, J\right)$.

\begin{tabular}{lcc}
\hline \hline Decay & $J=1 / 2$ & $J=3 / 2$ \\
\hline$\Xi_{c}^{3 / 2}\left(\overline{\mathbf{1 5}}_{1}, J\right) \rightarrow \Xi_{c}\left(\overline{\mathbf{3}}_{0}, 1 / 2\right)+\pi$ & 1.67 & 2.49 \\
$\Xi_{c}^{3 / 2}\left(\overline{\mathbf{1 5}}_{1}, J\right) \rightarrow \Xi_{c}\left(\mathbf{6}_{1}, 1 / 2\right)+\pi$ & 0.030 & 0.013 \\
$\Xi_{c}^{3 / 2}\left(\overline{\mathbf{1 5}}_{1}, J\right) \rightarrow \Xi_{c}\left(\mathbf{6}_{1}, 3 / 2\right)+\pi$ & 0.007 & 0.038 \\
$\Xi_{c}^{3 / 2}\left(\overline{\mathbf{1 5}}_{1}, J\right) \rightarrow \Sigma_{c}\left(\mathbf{6}_{1}, 1 / 2\right)+K$ & $\cdots$ & 0.003 \\
Total & 1.71 & 2.54 \\
\hline \hline
\end{tabular}




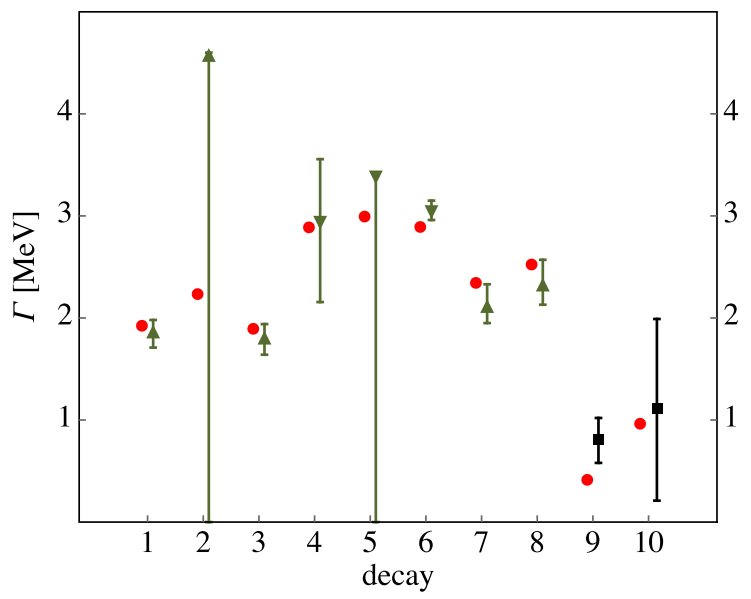

FIG. 1. Decay widths of the charm baryons. Numbers on the horizontal axis label the decay modes as listed in Table I of the article and Tables I and II. Red full circles correspond to our theoretical predictions. Dark green triangles correspond to the experimental data [1]. Data for decays 4-7 of $\Sigma_{c}\left(\mathbf{6}_{1}, 3 / 2\right)$ (down triangles) have been divided by a factor of 5 to fit within the plot area. Widths of two LHCb [2] $\Omega_{c}$ states that we interpret as pentaquarks are plotted as black full squares with corrected theoretical values shown as red full circles.

\section{APPENDIX: CORRECTION FACTORS $\gamma$ FOR SPIN STATES}

In this appendix we show how to calculate squares of the amplitudes with summation over spins. For definiteness we concentrate on an operator $\mathcal{O}_{\varphi}=D_{\varphi m}^{(8)} p_{m}$. We need

$$
\begin{aligned}
\mathcal{A}_{\varphi m}= & \left\langle B_{2}^{\mathcal{R}_{2}} J_{2} M_{2}\left|D_{\varphi m}^{(8)}\right| B_{1}^{\mathcal{R}_{1}} J_{1} M_{1}\right\rangle \\
= & \sum_{M, m_{1}, m_{2}}\left(\begin{array}{cc|c}
s_{1} & 1 / 2 & J_{1} \\
m_{1} & M & M_{1}
\end{array}\right)\left(\begin{array}{cc|c}
s_{2} & 1 / 2 & J_{2} \\
m_{2} & M & M_{2}
\end{array}\right) \\
& \times\left\langle\left(\mathcal{R}_{2}\right) B_{2},\left(-Y^{\prime}, s_{2}, m_{2}\right)\left|D_{\varphi m}^{(8)}\right|\left(\mathcal{R}_{1}\right) B_{1},\left(-Y^{\prime}, s_{1}, m_{1}\right)\right\rangle,
\end{aligned}
$$

where we have used that

$$
\left\langle 1 / 2, M^{\prime} \mid 1 / 2, M\right\rangle=\delta_{M^{\prime} M}
$$

The corresponding wave function of the light sector is given in terms of the Wigner rotational $D(\Omega)$ matrices,

$$
\Psi_{\left(B ;-Y^{\prime}, s, m\right)}^{(\mathcal{R})}(\Omega)=\left\langle\Omega \mid(\mathcal{R}) B,\left(-Y^{\prime}, s, m\right)\right\rangle=\sqrt{\operatorname{dim}(\mathcal{R})}(-)^{m-Y^{\prime} / 2} D_{\left(Y, T, T_{3}\right)\left(Y^{\prime}, s,-m\right)}^{(\mathcal{R}) *}(\Omega) .
$$

For the heavy baryons $Y^{\prime}=2 / 3 . \Omega(t)$ denotes relative configuration space- $\mathrm{SU}(3)$ group space rotation matrix.

The $D^{(8)}$ function matrix element reads as follows:

$$
\begin{aligned}
& \left\langle\left(\mathcal{R}_{2}\right) B_{2},\left(-Y^{\prime}, s_{2}, m_{2}\right)\left|D_{\varphi m}^{(8)}\right|\left(\mathcal{R}_{1}\right) B_{1},\left(-Y^{\prime}, s_{1}, m_{1}\right)\right\rangle \\
& =\sqrt{\frac{\operatorname{dim} \mathcal{R}_{2}}{\operatorname{dim} \mathcal{R}_{1}}}(-)^{m_{1}+m_{2}}\left(\begin{array}{cc|c}
8 & \mathcal{R}_{2} & \mathcal{R}_{1} \\
\varphi & B_{2} & B_{1}
\end{array}\right)\left(\begin{array}{cc|c}
8 & \mathcal{R}_{2} & \mathcal{R}_{1} \\
01 m & Y^{\prime} s_{2}-m_{2} & Y^{\prime} s_{1}-m_{1}
\end{array}\right) .
\end{aligned}
$$

We now decompose SU(3) Clebsch-Gordan coefficients into the SU(2) "clebsches" and the SU(3) isoscalar factors (in square brackets), 


$$
\begin{aligned}
\left(\begin{array}{cc|c}
8 & \mathcal{R}_{2} & \mathcal{R}_{1} \\
\varphi & B_{2} & B_{1}
\end{array}\right) & =\left(\begin{array}{cc|c|c}
T^{(\varphi)} & T^{(2)} & T^{(1)} \\
T_{3}^{(\varphi)} & T_{3}^{(2)} & T_{3}^{(1)}
\end{array}\right)\left[\begin{array}{cc|c}
8 & \mathcal{R}_{2} & \mathcal{R}_{1} \\
\varphi & B_{2} & B_{1}
\end{array}\right], \\
\left(\begin{array}{cc|c}
8 & \mathcal{R}_{2} & \mathcal{R}_{1} \\
01 m & Y^{\prime} s_{2}-m_{2} & Y^{\prime} s_{1}-m_{1}
\end{array}\right) & =\left(\begin{array}{ccc|c}
1 & s_{2} & s_{1} \\
m & -m_{2} & -m_{1}
\end{array}\right)\left[\begin{array}{ccc}
8 & \mathcal{R}_{2} & \mathcal{R}_{1} \\
01 & Y^{\prime} s_{2} & Y^{\prime} s_{1}
\end{array}\right] .
\end{aligned}
$$

Let us define amplitude $\mathcal{B}_{m}$ as follows:

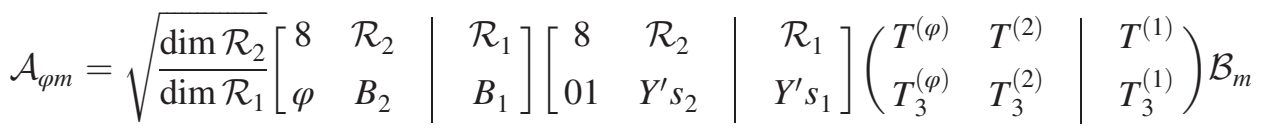

with

$$
\mathcal{B}_{m}=\sum_{M, m_{1}, m_{2}}(-)^{m_{1}+m_{2}}\left(\begin{array}{cc|c}
s_{1} & 1 / 2 & J_{1} \\
m_{1} & M & M_{1}
\end{array}\right)\left(\begin{array}{cc|c|c}
s_{2} & 1 / 2 & J_{2} \\
m_{2} & M & M_{2}
\end{array}\right)\left(\begin{array}{ccc}
1 & s_{2} & s_{1} \\
m & -m_{2} & -m_{1}
\end{array}\right) .
$$

While squaring the amplitude we average over initial baryon spin $M_{1}$ and sum over the final one $M_{2}$ (without summation over $J_{2}$ ),

$$
\mathcal{C}_{m^{\prime} m}\left(s_{1}, J_{1} ; s_{2}, J_{2}\right)=\frac{1}{2 J_{1}+1} \sum_{M_{1}, M_{2}} \mathcal{B}_{m^{\prime}} \mathcal{B}_{m}=\mathcal{C}\left(s_{1}, J_{1} ; s_{2}, J_{2}\right) \delta_{m^{\prime} m},
$$

where the last equality has to be proven, and we need to calculate $\mathcal{C}$.

Note that due to $m$ conservation we have

$$
m_{1}+M=M_{1}, \quad m_{2}+M=M_{2} \rightarrow m_{1}=M_{1}-M, \quad m_{2}=M_{2}-M
$$

so we can get rid of the summation over $m_{1}$ and $m_{2}$, and

$$
(-)^{m_{1}+m_{2}}=(-)^{M_{1}+M_{2}}(-)^{-2 M}=-(-)^{M_{1}+M_{2}} \text {. }
$$

Note that always $(-)^{-2 M}=-1$, because $M= \pm 1 / 2$. Hence

$$
\mathcal{B}_{m}=-(-)^{M_{1}+M_{2}} \sum_{M=-1 / 2}^{1 / 2}\left(\begin{array}{cc|c}
s_{1} & 1 / 2 & J_{1} \\
M_{1}-M & M & M_{1}
\end{array}\right)\left(\begin{array}{cc|c}
s_{2} & 1 / 2 & J_{2} \\
M_{2}-M & M & M_{2}
\end{array}\right)\left(\begin{array}{ccc}
1 & s_{2} & s_{1} \\
m & M-M_{2} & M-M_{1}
\end{array}\right) .
$$

Therefore we have finally

$$
\begin{aligned}
\mathcal{C}_{m^{\prime} m}= & \mathcal{C}_{m^{\prime} m}\left(s_{1}, J_{1} ; s_{2}, J_{2}\right) \\
= & \frac{1}{2 J_{1}+1} \sum_{M_{1}=-J_{1}}^{J_{1}} \sum_{M_{2}=-J_{2}}^{J_{2}} \\
& \times \sum_{M^{\prime}=-1 / 2}^{1 / 2}\left(\begin{array}{cc|c|c|c}
s_{1} & 1 / 2 & J_{1} \\
M_{1}-M^{\prime} & M^{\prime} & M_{1}
\end{array}\right)\left(\begin{array}{cc|c}
s_{2} & 1 / 2 & J_{2} \\
M_{2}-M^{\prime} & M^{\prime} & M_{2}
\end{array}\right)\left(\begin{array}{ccc}
1 & s_{2} & s_{1} \\
m^{\prime} & M^{\prime}-M_{2} & M^{\prime}-M_{1}
\end{array}\right) \\
& \times \sum_{M=-1 / 2}^{1 / 2}\left(\begin{array}{ccc}
s_{1} & 1 / 2 & J_{1} \\
M_{1}-M & M & M_{1}
\end{array}\right)\left(\begin{array}{ccc}
s_{2} & 1 / 2 & J_{2} \\
M_{2}-M & M & M_{2}
\end{array}\right)\left(\begin{array}{ccc}
1 & s_{2} & s_{1} \\
m & M-M_{2} & M-M_{1}
\end{array}\right),
\end{aligned}
$$

where we have used

$$
(-)^{2\left(M_{1}+M_{2}\right)}=1
$$


since $M_{1,2}$ are integers. Coefficients $\mathcal{C}_{m^{\prime} m}$ can be calculated numerically for the cases that we need for heavy baryon decays. They are indeed proportional to $\delta_{m^{\prime} m}$. The results are presented below.

\begin{tabular}{|c|c|c|c|c|c|}
\hline $\begin{array}{c}1- \\
s_{\text {soliton }}\end{array}$ & $\begin{array}{l}\text { initial } \\
J_{\text {baryon }} \\
\end{array}$ & $s_{\text {soliton }}$ & $\begin{array}{l}\text { final } \\
J_{\text {baryon }} \\
\end{array}$ & $\mathcal{C}$ & comments \\
\hline 1 & $1 / 2$ & 0 & $1 / 2$ & $1 / 3$ & $\begin{array}{c}\overline{\mathbf{1 5}}_{1}^{1 / 2} \rightarrow \overline{\mathbf{3}}_{0}^{1 / 2} \\
\mathbf{6}_{1}^{1 / 2} \rightarrow \overline{\mathbf{3}}_{0}^{1 / 2}\end{array}$ \\
\hline 1 & $3 / 2$ & 0 & $1 / 2$ & $1 / 3$ & $\begin{array}{c}\overline{\mathbf{1 5}}_{1}^{3 / 2} \rightarrow \overline{\mathbf{3}}_{0}^{1 / 2} \\
\mathbf{6}_{1}^{3 / 2} \rightarrow \overline{\mathbf{3}}_{0}^{1 / 2}\end{array}$ \\
\hline 1 & $1 / 2$ & 1 & $1 / 2$ & $2 / 9$ & $\begin{array}{c}\overline{\mathbf{1 5}}_{1}^{1 / 2} \rightarrow \mathbf{6}_{1}^{1 / 2} \\
\mathbf{6}_{1}^{1 / 2} \rightarrow \mathbf{6}_{1}^{1 / 2}\end{array}$ \\
\hline 1 & $1 / 2$ & 1 & $3 / 2$ & $1 / 9$ & $\begin{array}{c}\overline{\mathbf{1 5}}_{1}^{1 / 2} \rightarrow \mathbf{6}_{1}^{3 / 2} \\
\mathbf{6}_{1}^{1 / 2} \rightarrow \mathbf{6}_{1}^{3 / 2}\end{array}$ \\
\hline 1 & $3 / 2$ & 1 & $1 / 2$ & $1 / 18$ & $\begin{array}{c}\overline{\mathbf{1 5}}_{1}^{3 / 2} \rightarrow \mathbf{6}_{1}^{1 / 2} \\
\mathbf{6}_{1}^{3 / 2} \rightarrow \mathbf{6}_{1}^{1 / 2}\end{array}$ \\
\hline 1 & $3 / 2$ & 1 & $3 / 2$ & $5 / 18$ & $\begin{aligned} \overline{\mathbf{1 5}}_{1}^{3 / 2} & \rightarrow \mathbf{6}_{1}^{3 / 2} \\
\mathbf{6}_{1}^{3 / 2} & \rightarrow \mathbf{6}_{1}^{3 / 2}\end{aligned}$ \\
\hline
\end{tabular}

Note that

$$
\sum_{J_{\text {final }}} \mathcal{C}\left(J_{\text {initial }} \rightarrow J_{\text {final }}\right)=\frac{1}{3}
$$

Correction factors $\gamma$ are defined as $\gamma=3 \mathcal{C}$.

Finally we can sum and average over the isospin with the help of the following identity:

$$
\frac{1}{2 T^{(1)}+1} \sum_{T_{3}^{(\varphi)}, T_{3}^{(2)}, T_{3}^{(1)}}\left(\begin{array}{cc|c}
T^{(\varphi)} & T^{(2)} & T^{(1)} \\
T_{3}^{(\varphi)} & T_{3}^{(2)} & T_{3}^{(1)}
\end{array}\right)^{2}=1 .
$$

If initial and final states are known, the relevant isospin Clebsch-Gordan coefficient squared has to be included.

Finally we have

$$
\begin{aligned}
\overline{\mathcal{A}_{\varphi}^{2}} & =\frac{1}{2 T^{(1)}+1} \sum_{T_{3}^{(\varphi)}, T_{3}^{(2)}, T_{3}^{(1)}} \frac{1}{2 J_{1}+1} \sum_{M_{1}, M_{2}} \mathcal{A}_{\varphi m^{\prime}} \mathcal{A}_{\varphi m} p_{m^{\prime}} p_{m} \\
& =\frac{\operatorname{dim} \mathcal{R}_{2}}{\operatorname{dim} \mathcal{R}_{1}}\left[\begin{array}{cc|c|c}
8 & \mathcal{R}_{2} & \mathcal{R}_{1} \\
\varphi & B_{2} & B_{1}
\end{array}\right]^{2}\left[\begin{array}{ccc}
8 & \mathcal{R}_{2} & \mathcal{R}_{1} \\
01 & Y^{\prime} s_{2} & Y^{\prime} s_{1}
\end{array}\right]^{2} \mathcal{C}\left(s_{1}, J_{1} ; s_{2}, J_{2}\right) p^{2} .
\end{aligned}
$$


We have checked explicitly that the spin summation factors are identical for the remaining two terms of the decay operator.

[1] C. Patrignani et al. (Particle Data Group Collaboration), Chin. Phys. C 40, 100001 (2016).

[2] R. Aaij et al. (LHCb Collaboration), Phys. Rev. Lett. 118, 182001 (2017). 